\title{
Les porphyries héréditaires: de la biochimie clinique à la pathologie moléculaire
}

L'identification des lésions biochimiques des porphyries est aujourd'hui complète. C'est à l'analyse fine des gènes codant pour les protéines en cause et de leurs anomalies que s'attaque la recherche, en attendant d'entreprendre la thérapeutique, éventuellement génétique, de ces affections.

\section{Hubert de Verneuil Bernard Grandchamp Jean-Charles Deybach Carole Beaumont Yves Nordmann}

\footnotetext{
ADRESSES

Service de Biochimie, Hôpital Louis-Mourier, 178 rue des Renouillers, 92701 Colombes Cedex.

Laboratoire de génétique moléculaire, faculté de médecine Xavier-Bichat, 16 rue HenriHuchard, 75018 Paris.

$m / s n^{\circ} 3$ ool. 3 , mars 87
}

es porphyries représentent un ensemble de maladies métaboliques caractérisées par une synthèse, une accumulation et une excrétion accrues de porphyrines et/ou de leurs précurseurs, acide delta aminolévulinique et porphobilinogène (figure 1).

Elles sont classiquement regroupées en porphyries hépatiques et porphyries érythropoïétiques selon le tissu dans lequel prédomine le trouble métabolique (Tableau I). Chaque type de porphyrie présente un profil d'excrétion spécifique durant les crises tandis que les manifestations cliniques sont polymorphes et souvent non caractéristiques. Depuis les années 1970 , des progrès très importants ont été réalisés dans la connaissance de ces maladies puisque chaque porphyrie a pu être reliée à un déficit enzymatique d'une des enzymes de la biosynthèse de l'hème $[1,2]$ (Tableau II). Les différentes manifestations cliniques des porphyries (signes cutanés, abdominaux, neurologiques) ainsi qu'une coloration rouge-porto des urines conduisent à demander un dosage des porphyrines et de leurs précurseurs dans les différents milieux biologiques (urines, sang et selles). Le dosage de l'activité de l'enzyme spécifiquement déficitaire permet de confirmer le type de la porphyrie et d'engager une enquête familiale : la recherche des porteurs asymptomatiques est en effet fondamentale pour assurer la prévention des accidents liés à la prise de certains médicaments dits " porphyrinogéniques". S'agissant pour la plupart de maladies dominantes à pénétrance faible (un sujet porteur du déficit enzymatique sur dix développera la maladie), leur fréquence est très difficile à évaluer : en France, la porphyrie aiguë intermittente et la porphyrie cutanée prédominent largement.

L'hétérogénéité des mutations au sein des différents types de maladies a pu être démontrée aussi bien par l'étude des propriétés cinétiques et du " turnover " des différentes enzymes que par la mise en évidence ou non d'une protéine immunoréactive dépour- 


\section{RÉFÉRENCES}

1. Kappas A, Sassa S, Anderson KE. The Porphyrias. In : Stanbury JB, Wyngaarden DS, Frederickson DS, Goldstein JL, Brown MS, eds. The Metabolic Basis of Inherited Disease. New York : Mc Graw Hill, 1983 : 1301-84.

2. Elder GH. Metabolic abnormalities in the porphyrias. Seminars in Dermatology 1986 ; 5 : 88-98.

3. Sassa S. Sequential induction of heme pathway enzymes during erythroid differentiation of mouse Friend Leukemia virus-infected cells. $J$ Exp Med 1976 ; 143 : 305-10.

4. Beaumont C, Deybach JC, Grandchamp B, Da Silva V, de Verneuil H, Nordmann Y. Effects of succinylacetone on dimethylsulfoxidemediated induction of heme pathway enzymes in mouse Friend virus-transformed erythroleukemia cells. Exp Cell Res 1984 ; 154 : 474-84.

5. Borthwick IA, Srivastava G, Day AR, et al. Complete nucleotide sequence of hepatic 5 aminolevulinate synthase precursor. Eur J Biochem 1985 ; 150 : 481-4.

6. Yamamoto M, Yew NS, Federspiel M, Dodgson JB, Hayashi N, Engel JD. Isolation of recombinant cDNAs encoding chicken erythroid delta aminolevulinate synthase. Proc Natl Acad Sci USA 1985 ; 82 : 3702-6.

7. Maguire DJ, Day AR, Borthwick IA, et al. Nucleotide sequence of the chicken 5 aminolevulinate synthase gene. Nucleic Acid Res 1986 ; 14 : 1379-91.

8. Wetmur JG, Bishop DF, Cantelmo C, Desnick RJ. Human delta aminolevulinate dehydratase : nucleotide sequence of a full-length cDNA clone. Proc Natl Acad Sci USA 1986 ; 83 : 7703-7.

9. Grandchamp B, Roméo PH, Dubart A, et al. Molecular cloning of a cDNA sequence complementary to porphobilinogen deaminase mRNA from rat. Proc Natl Acad Sci USA 1984 ; 81 : 5036-40.

10. Raich N, Roméo PH, Dubart A, Beaupain D, Cohen-Solal M, Goossens M. Molecular cloning and complete primary sequence of human erythrocyte porphobilinogen deaminase. Nucleic Acid Res 1986 ; 14 : 5955-67.

11. Grandchamp B, de Verneuil H, Beaumont C, Chrétien S, Walter O, Nordmann Y. Tissue-specific expression of porphobilinogen deaminase : two isoenzymes from a single gene. Eur J Biochem 1986 (sous presse).

12. Roméo PH, Dubart A, Grandchamp B, et al. Isolation and identification of a cDNA clone coding for rat uroporphyrinogen decarboxylase.

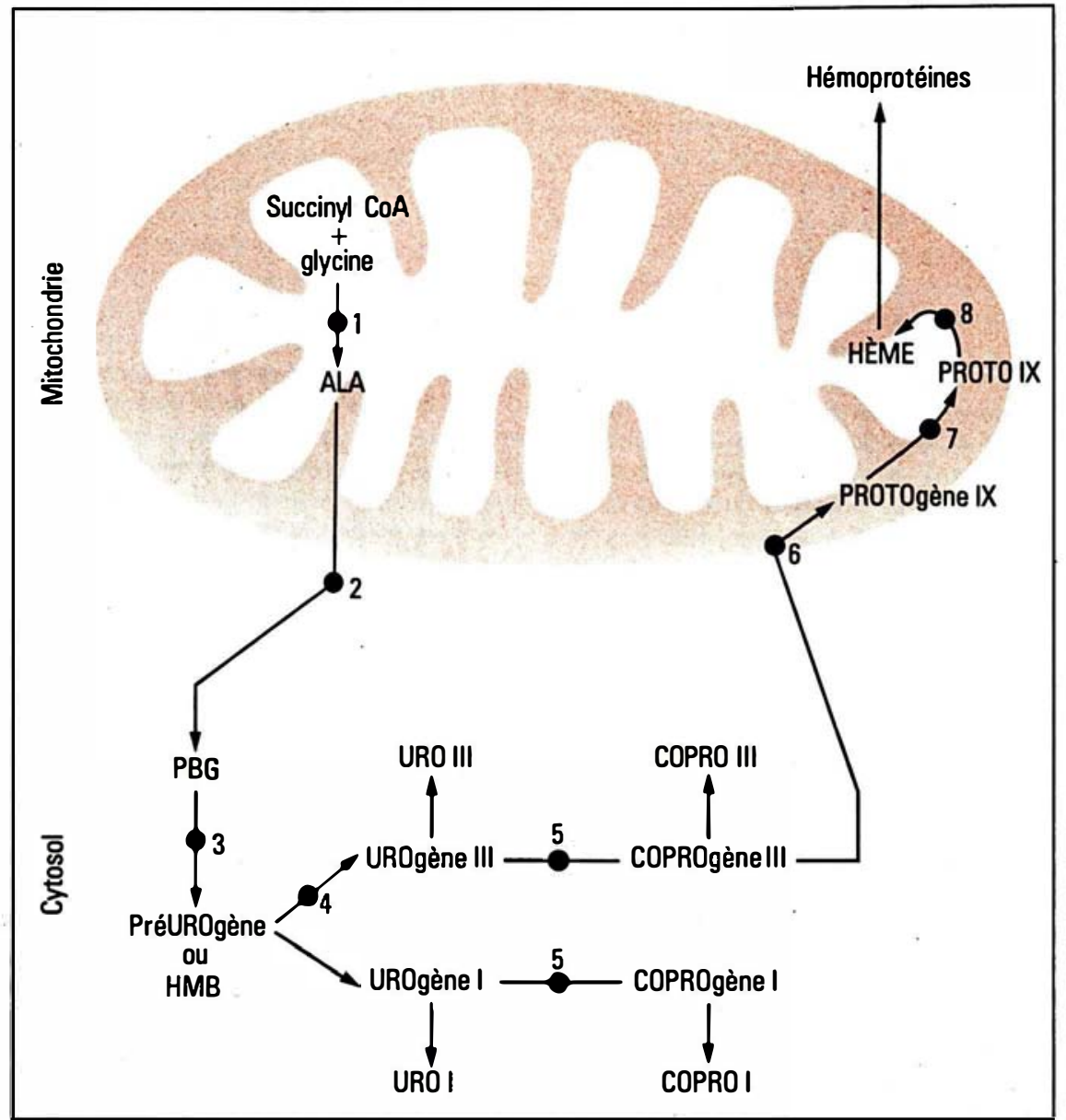

Figure 1. Biosynthèse de l'hème : différentes étapes et compartimentation cellulaire. ALA : acide $\delta$ aminolévulinique. PBG : porphobilinogène. HMB : hydroxyméthylbilane. URO : uroporphyrine. UROgène : uroporphyrinogène. COPRO : coproporphyrine. COPROgène : coproporphyrinogène. PROTO : protoporphyrine. PROTOgène : protoporphyrinogene.

Enzymes cytosoliques : 2. Aminolévulinate déshydratase. 3. Porphobilinogène désaminase ou hydroxyméthylbilane synthétase. 4. Uroporphyrinogène III cosynthétase. 5. Uroporphyrinogène décarboxylase.

Enzymes mitochondriales : 1. Aminolévulinate synthétase : matrice mitochondriale. 6. Coproporphyrinogène oxydase : espace intermembranaire. 7. Protoporphyrinogène oxydase : membrane interne. 8. Ferrochélatase : membrane interne, face matricielle.

\section{ABRÉVIATIONS}

$A L A$ : Acide $\delta$ aminolévulinique

$H M B$ : Hydroxyméthylbilane

COPRO : Coproporphyrine

COPROgène : Coproporphyrinogène

$P B G$ : Porphobilinogène

PROTO : Protoporphyrine
PROTOgène : Protoporphyrinogène URO : Uroporphyrine UROgène : Uroporphyrinogène PAI : Porphyrie aiguë intermittente PCT : Porphyrie cutanée tardive 
vue d'activité enzymatique. Le développement de la biologie moléculaire permet de poursuivre ces études directement au niveau de l'ADN.

\section{Biosynthèse de l'hème : les gènes}

La voie métabolique de biosynthèse de l'hème est identique dans toutes les cellules de mammifères. A l'exception de l'acide delta aminolévulinique (ALA) synthétase (dont le déficit enzymatique entraîne un type d'anémie sidéroblastique et non une porphyrie), il existe des arguments génétiques tendant à démontrer que chacune des enzymes impliquées dans cette chaîne métabolique est codée par un même gène dans les différents types cellulaires : en effet, pour une porphyrie donnée, le déficit enzymatique a été retrouvé dans tous les tissus étudiés.

Localisation chromosomique. Quatre parmi les gènes codant pour des enzymes de la synthèse de l'hème ont été localisés sur différents chromosomes humains : l'aminolévulinate (ALA) déshydra- tase sur le chromosome 9 ; la porphobilinogène (PBG) désaminase sur le chromosome 11; l'uroporphyrinogène (URO) décarboxylase sur le chromosome 1 (région p34) et la coproporphyrinogène oxydase sur le chromosome 9. Ainsi ces enzymes qui sont fonctionnellement associées ne sont pas codées par des gènes regroupés sur le génome. Ce point est spécialement intéressant à étudier car l'on sait qu'il existe une induction coordonnée de ces enzymes au cours de la différenciation érythropoïétique $[3,4]$. Il est raisonnable de penser que cette expression coordonnée doit être sous la dépendance de gènes de régulation (agissant par l'intermédiaire de facteurs diffusibles se fixant à l'ADN), plutôt que sous la dépendance directe des gènes de structure.

ADN complémentaires et/ou gènes clonés. L'étude moléculaire des gènes codant pour les enzymes de la biosynthèse de l'hème a débuté pour 4 d'entre eux :

- L'ALA synthétose. Chez les mammiferes et les oiseaux, l'ALA synthétase hépatique differe de

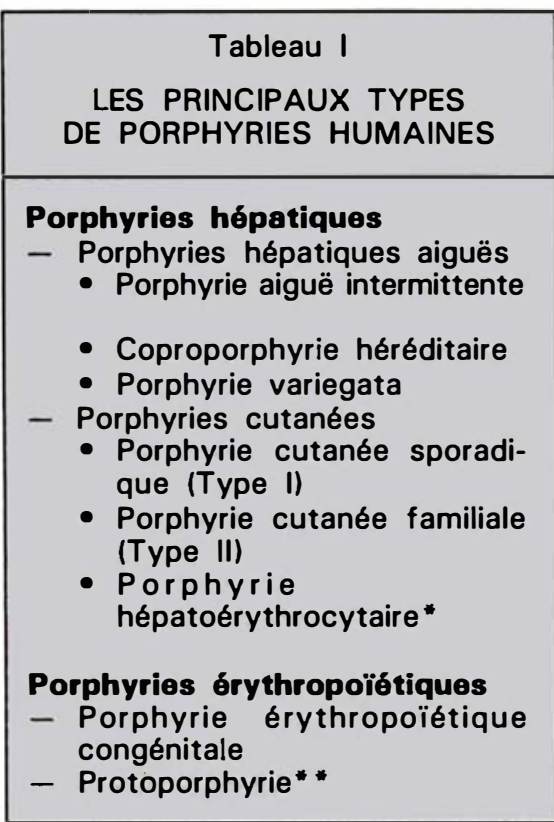

- Porphyrie dans laquelle le trouble métabolique s'exprime aussi dans le système énthropoïétique. *- Porphyrie dans laquelle le trouble métabolique s'exprime aussi dans le foie.

\begin{tabular}{|c|c|c|c|}
\hline \multicolumn{4}{|c|}{ PORPHYRIES HUMAINES : MODE DE TRANSMISSION ET DÉFICITS ENZYMATIQUES } \\
\hline Maladie & $\begin{array}{l}\text { Mode de } \\
\text { transmission }\end{array}$ & $\begin{array}{l}\text { Enzyme déficitaire : } \\
\text { activité par rapport } \\
\text { aux contrôles }\end{array}$ & $\begin{array}{l}\text { Organes dans lesquels } \\
\text { le déficit a été démontré }\end{array}$ \\
\hline Porphyrie aiguë intermittente & $\begin{array}{l}\text { autosomique } \\
\text { dominant }\end{array}$ & PBG désaminase : $50 \%$ & $\begin{array}{c}\text { Foie, érythrocytes, } \\
\text { fibroblastes, cellules } \\
\text { amniotiques, lymphoblastes }\end{array}$ \\
\hline Coproporphyrie & $\begin{array}{l}\text { autosomique } \\
\text { dominant }\end{array}$ & $\begin{array}{c}\text { COPROgène } \\
\text { oxydase : } 50 \%\end{array}$ & $\begin{array}{l}\text { Fibroblastes, foie, } \\
\text { lymphocytes }\end{array}$ \\
\hline Porphyrie variegata & $\begin{array}{l}\text { autosomique } \\
\text { dominant }\end{array}$ & $\begin{array}{c}\text { PROTOgène } \\
\text { oxydase : } 50 \%\end{array}$ & $\begin{array}{c}\text { Cellules de la moelle osseuse, } \\
\text { fibroblastes, lymphocytes }\end{array}$ \\
\hline Porphyrie type 1: sporadique & $?$ & $\begin{array}{c}\text { UROgène } \\
\text { décarboxylase : } 50 \% \\
\end{array}$ & Foie uniquement \\
\hline cutanée type II : familial & $\begin{array}{c}\text { autosomique } \\
\text { dominant }\end{array}$ & $\begin{array}{c}\text { UROgène } \\
\text { décarboxylase : } 50 \%\end{array}$ & $\begin{array}{c}\text { Erythrocytes, foie, } \\
\text { lymphocytes, fibroblastes }\end{array}$ \\
\hline Porphyrie hépatoérythrocytaire & $\begin{array}{l}\text { autosomique } \\
\text { récessif ? }\end{array}$ & $\begin{array}{c}\text { UROgène } \\
\text { décarboxylase : } 5-14 \% \\
\end{array}$ & $\begin{array}{c}\text { Erythrocytes, lymphocytes, } \\
\text { fibroblastes }\end{array}$ \\
\hline Protoporphyrie & $\begin{array}{l}\text { autosomique } \\
\text { dominant }\end{array}$ & Ferrochélatase : 14-35 \% & $\begin{array}{l}\text { Moelle osseuse, foie, } \\
\text { fibroblastes, sang, } \\
\text { lymphocytes }\end{array}$ \\
\hline $\begin{array}{l}\text { Porphyrie érythropoïétique } \\
\text { congénitale }\end{array}$ & $\begin{array}{l}\text { autosomique } \\
\text { récessif }\end{array}$ & $\begin{array}{c}\text { UROgène III } \\
\text { cosynthétase : } 2-25 \%\end{array}$ & $\begin{array}{l}\text { Erythrocytes, } \\
\text { fibroblastes }\end{array}$ \\
\hline
\end{tabular}




\section{RÉFÉRENCES}

13. Roméo PH, Raich N, Dubart A, et al. Molecular cloning and nucleotide sequence of a complete human uroporphyrinogen decarboxylase cDNA. J Biol Chan 1986; 261 : 9825-31.

14. Borthwick IA, Srivastava G, Hobbs AA, et al. Cellular Regulation and Malignant Growth. In : Ebashi S, ed. Tokyo: Springer Verlag (Berlin), 1985 : 144-51.

15. Ponka P, Schulman HM. Regulation of heme synthesis in erythroid cells by iron delivery from transferrin. In : Nordmann Y, ed. Porphyrins and Porphyrios. Colloque Inserm/John Libbey Eurotext 1986 ; 134 : 55-67.

16. Mustajoki P. Normal erythrocyte uroporphyrinogen I synthase in a kindred with acute intermittent porphyria. Ann Intern Med $1981 ; 95: 162-6$.

17. De Verneuil H, Aitken G, Nordmann Y. Familial and sporadic porphyria cutanea tarda : two different diseases. Hum Genct 1978 ; $44: 145-51$.

18. Pinol Aguadé J, Castells A, Indacochea A, Rodes J. A case of biochemically unclassifiable hepatic porphyria. Br J Dermatol $1969 ; 81$ : 270-4.

19. Elder GH, Smith SG, Herrero C, et al. Hepato-erythropoietic : a new uroporphyrinogen decarboxylase defect or homozygous porphyria cutanea tarda ? Lancet 1981 ; i : 916-9.

20. De Verneuil H, Beaumont C, Deybach JC, Nordmann Y, Sfar Z, Kastally R. Enzymatic and immunological studies of uroporphyrinogen decarboxylase in familial porphyria cutanea tarda and hepatoerythropoietic porphyria. Am J Hum Genet 1984 ; 36 : 613-22.

21. Desnick RJ, Ostasiewicz LT, Tishler PA, Mustajoki P. Acute intermittent porphyria : characterization of a novel mutation in the structural gene of porphobilinogen deaminase. $J$ Clin Invest 1985; 76 : 865-74.

22. Elder GH, Urquhart AJ, De Salamanca RE, Munoz JJ, Bonkowsky HL. Immunoreactive uroporphyrinogen decarboxylase in liver in porphyria cutanea tarda. Lancet 1985 ; i : 229-33.

23. De Verneuil H, Grandchamp B, Beaumont C, Picat C, Nordmann Y. Uroporphyrinogen decarboxylase structural mutant (Gly 281 - Glu) in a case of porphyria. Science $1986 ; 234$ : 732-4.

24. Belmont JW, Henrel-Tigges J, Chang SMW, et al. Expression of human adenosine deaminase in murine haematopoietic progenitor cells following retroviral transfer. Nature $1986 ; 322: 385-7$. l'enzyme présente dans le tissu érythropoïétique et il est clairement établi que les deux enzymes résultent de la traduction de deux ARNm différents. L'analyse des ADN complémentaires des messagers érythropoïétiques et hépatiques $[5,6]$ suggère fortement l'existence d'au moins deux gènes distincts dont les séquences nucléotidiques ne présentent pas d'hybridation croisée [7]. Ce problème est intéressant d'un point de vue fondamental pour l'étude de la régulation de l'expression génétique et aussi d'un point de vue appliqué pour l'étude du déficit enzymatique en ALA synthétase retrouvé dans les érythroblastes de certaines formes d'anémie sidéroblastique congénitale.

- L'ALA déshydratase. Le clonage d'un ADN complémentaire de l'ARN messager de l'ALA déshydratase humaine a été récemment rapporté [8]. La différence de séquence nucléotidique entre plusieurs clones est peut-être à la base du polymorphisme génétique trouvé au niveau de la protéine (isoenzymes de charge différente). - La PBG désaminase. L'étude moléculaire de la PBG désaminase a débuté par le clonage et la caractérisation d'un ADN complémentaire de l'ARNm de rate érythropoïétique de rat [19]. Des clones ADN complémentaires des ARN messagers humains de type érythropoiétique et non érythropoïétique ont été ensuite isolés
$[10,11]$. La comparaison des séquences protéiques déduites des séquences nucléotidiques indique que la PBG désaminase de cellules non érythropoïétiques differe de la PBG désaminase érythropoiétique par la présence d'un peptide de 17 acides aminés à l'extrémité $\mathrm{NH}_{2}$ terminale [11]. Les résultats déjà obtenus par notre groupe et le groupe de Michel Goossens à Crêteil (Unité Inserm U.91) permettent de proposer le modèle d'expression génétique suivant : le gène de la PBG désaminase contiendrait deux régions promotrices ( $\mathrm{P} 1$ et $\mathrm{P} 2)$ et chacune de ces régions serait spécifiquement utilisée pour l'initiation de la transcription (P1 dans la plupart des types cellulaires et P2, seulement dans les cellules érythropoïétiques, à partir d'un certain stade de maturation) ; un épissage différent, dépendant du promoteur utilisé, conduirait à des ARNm de structure AC ou BC codant pour une PBG désaminase respectivement de type non érythropoiétique ou érythropoïétique (figure 2).

- L'URO décarboxylase. L'ADN complémentaire de l'ARN messager extrait de rate érythropoïétique a été cloné chez le rat [12] et chez l'homme [13]. Le gène est présent à l'état unique dans le génome humain et une seule et même espèce d'ARN a été mise en évidence dans les différents tissus. La plus grande abondance du

Figure 2. Los 2 ARNm do la PBG désamlnase. A : séquence spécifique de I'ARNm non érythropoïétique. B : séquence spécifique de l'ARNm érythropoïétique. C: séquence commune aux deux types d'ARNm. 


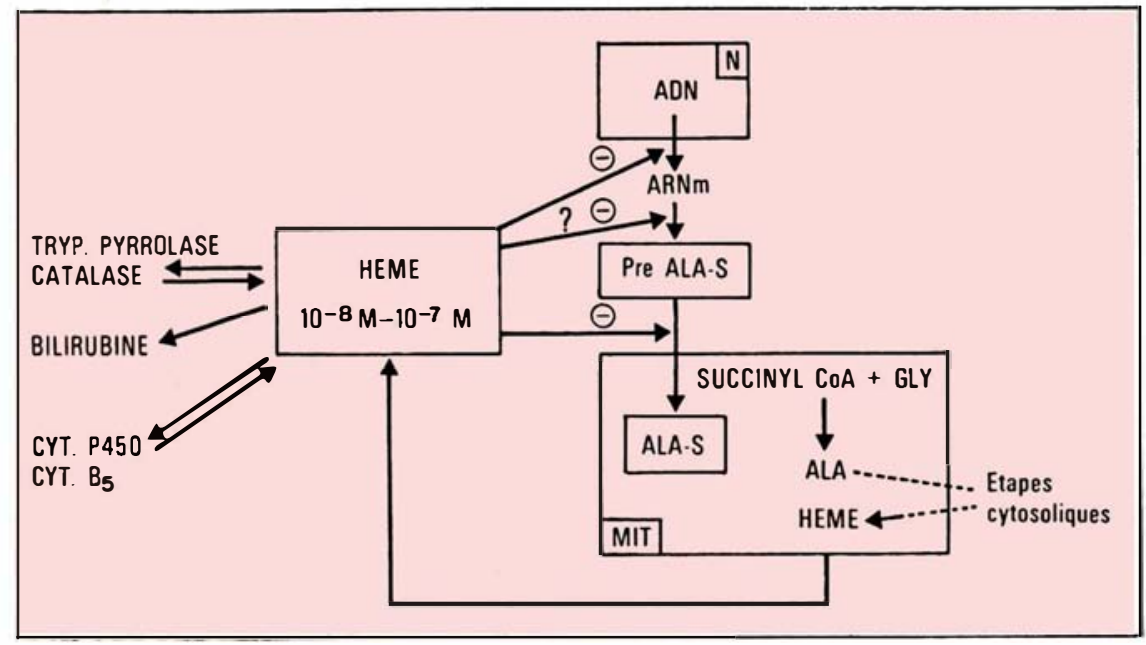

Figure 3. Régulation de I'ALA synthétase par I'hèmo dans lo foie. $N=$ Noyau ; MIT = Mitochondrie ; TRYP.PYRROLASE = Tryptophane pyrrolase. messager dans le tissu érythropoïétique est en relation avec une transcription plus importante du gène dans ce dernier tissu que dans les autres types cellulaires [13].

\section{Biosynthèse de l'hème : régulation}

Régulation de l'activité de l'ALA synthétase par l'hème. L'hème, produit final de la chaîne de biosynthèse, exerce un rétrocontrôle négatif sur l'ALA synthétase hépatique. L'inhibition de l'enzyme a été démontrée in vitro mais à des concentrations d'hème très élevées $\left(10^{-4} \mathrm{M}\right)$, ce qui n'a pas de signification sur le plan physiologique. La répression de la synthèse de l'ALA synthétase par l'hème est en revanche d'une importance fondamentale : c'est à des concentrations physiologiques $\left(10^{-8}\right.$ à $\left.10^{-} \mathrm{M}\right)$ que $l^{\prime}$ hème s'oppose à l'induction de l'ALA synthétase par certaines drogues. Le contrôle de la synthèse enzymatique peut se faire à différents niveaux : transcriptionnel, traductionnel, post-traductionnel. Le contrôle post-traductionnel a été mis en évidence par plusieurs groupes : l'hème inhibe la matu$m / s n^{\circ} 3$ ool. 3 , mars 87 l'activité de l'ALA synthétase. Cette augmentation est liée à une diminution de la concentration intracellulaire de l'hème libre et, par conséquent, une diminution de la répression de la synthèse de l'enzyme (figure 4). La décroissance de l'hème libre peut être liée à un blocage d'une (ou plusieurs) enzymes de la chaîne de biosynthèse (plomb, DDC, griséofulvine, succinylacétone), à une destruction de l'hème (AIA, apronalide), ou encore à une augmentation de l'utilisation de l'hème par stimulation de la synthèse de l'apoprotéine des hémoprotéines du groupe du cytochrome $\mathrm{P} 450$ : cette apoprotéine a une haute affinité pour l'hème et va donc contribuer à la diminution de l'hème libre. Le cytochrome P450 (cytochrome microsomal) joue un rôle majeur dans la "détoxification " par le foie de nombreuses drogues : sa concentration augmente lors de l'administration de ces drogues. Ces différentes considérations physiopathologiques sont à prendre en compte lors du traitement des crises de porphyrie : a) il faut s'assurer que les médicaments utilisés pour le traitement symptomatique ne sont pas des inducteurs ; b) l'hématine (dérivé de l'hème, produit final) qui est utilisée pour réprimer l'ALA synthétase entraîne une réduction rapide de l'accumulation des précurseurs et/ou de leurs dérivés avec pour conséquence une disparition progressive des signes neurologiques.

Régulation dans le système érythropoiétique. Un effet régulateur de l'hème sur l'ALA synthétase reste controversé dans le système érythropoïétique. Différents travaux suggèrent que la biosynthèse de l'hème n'est pas limitée par l'activité de l'ALA synthétase mais plutôt par une étape encore mal précisée du métabolisme cellulaire du fer [15]. Lors de la différenciation érythropoïétique, l'activité des enzymes de la chaîne de biosynthèse de l'hème augmente de façon précoce et coordonnée. Cette induction est la conséquence d'une augmentation de l'activité transcriptionnelle 


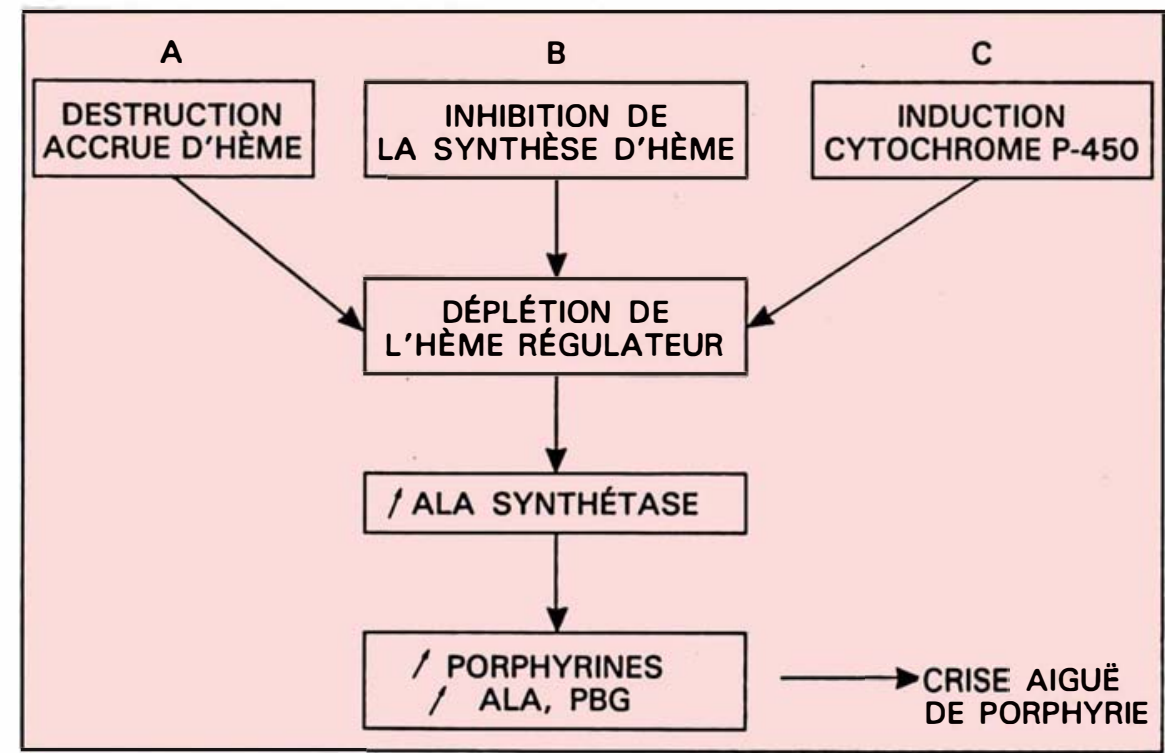

Figure 4. Porphyries expérimentales et mécanismes du déclenchement des crises aiguës dans les porphyries hépatiques. Une porphyrie expérimentale peut être réalisée par l'un des trois mécanismes $A, B$ ou $C$. Chez un individu porphyrique, l'association des mécanismes $B$ (par déficit enzymatique héréditaire) et $C$ (par prise de médicaments inducteurs comme les barbituriques) est le plus souvent responsable du déclenchement de la crise aiguë.

des gènes correspondants. De plus, l'hème joue un rôle dans la régulation de la synthèse des châ̂nes de globine, à la fois aux niveaux traductionnel et prétraductionnel.

\section{Porphyries aiguë inter- mittente et $P$. cutanée}

Porphyrie aiguë intermittente (PAI). La PAI se manifeste par la survenue de crises aiguës de la maladie : syndrome douloureux abdominal associé parfois à un syndrome neurologique et/ou psychiatrique [1]. Les erreurs d'orientation dans la recherche de leur étiologie peuvent conduire à de graves conséquences thérapeutiques, par exemple lors de l'emploi des barbituriques. La " crise" de porphyrie est confirmée par les dosages biologiques (notamment l'augmentation des précurseurs). et le type de la porphyrie affirmé par le dosage enzymatique (déficit en PBG désaminase dans ce cas). La maladie a une transmission autosomique dominante. Un cas particulier de PAI a été décrit où le défi- dans le système érythropoiétique, mais serait limité au foie et aux autres tissus non érythropoïétiques [16].

Porphyrie cutanée symptomatique (ou porphyrie cutanée tardive, PCT). Contrairement aux autres porphyries hépatiques, les manifestations cliniques de cette porphyrie sont essentiellement cutanées. Des données récentes permettent d'en distinguer deux types [17]: le type sporadique (type I) de survenue tardive, qui apparaît généralement en association avec d'autres désordres hépatiques (d'origine éthylique le plus souvent) ou avec la prise d'oestrogènes : l'activité de l'URO décarboxylase érythrocytaire est normale. Le déficit enzymatique est limité au foie; l'origine, héréditaire ou acquise, de la maladie n'a pu être encore clairement établie ; le type familial (type II) qui apparaît aussi bien chez les enfants que chez les adultes, sans désordre hépatique manifeste ; plusieurs cas peuvent être détectés dans une même famille; l'hérédité de la maladie se manifeste sur le mode autosomique dominant. L'activité de l'URO décarboxylase éry- throcytaire est abaissée de $50 \%$ par rapport à l'activité normale. Le déficit se retrouve dans toutes les cellules de l'organisme.

La porphyrie hépatoérythrocytaire (HEP) est une forme sévère de porphyrie qui associe des troubles hématologiques aux manifestations cutanées [18]. Le profil d'excrétion des porphyrines dans les urines et les selles est tout à fait similaire à celui rencontré dans la porphyrie cutanée. Dans tous les cas étudiés, un important déficit enzymatique en URO décarboxylase a pu être mis en évidence dans les érythrocytes, les lymphocytes ou les fibroblastes, compatible avec une transmission autosomique récessive de la maladie $[19,20]$. Bien que cela soit vraisemblable, il est néanmoins impossible de conclure que la porphyrie hépatoérythrocytaire est une forme homozygote de la porphyrie cutanée familiale : en effet, une porphyrie cutanée cliniquement manifeste n'a jamais à notre connaissance été décrite dans une famille comportant un ou plusieurs malades atteints de porphyrie hépatoérythrocytaire. L'analyse moléculaire des mutations responsables de ces différentes conditions permettra seule d'affirmer que la porphyrie hépatoérythrocytaire est caractérisée par un ensemble de mutations qui se différencient de la ou les mutations retrouvées dans la porphyrie cutanée familiale.

\section{Étude moléculaire du déficit enzymatique}

La porphyrie aiguë intermittente (déficit en PBG désaminase) ainsi que la porphyrie cutanée familiale et la porphyrie hépatoérythrocytaire (déficits en URO décarboxylase) sont les principales porphyries pour lesquelles la caractérisation moléculaire des mutations a débuté depuis quelques années. Porphyrie aiguë intermittente. L'étude de la protéine déficitaire à l'aide d'anticorps spécifiques a pu mettre en évidence une hétérogénéité des mutations dans la PAI [21]. En effet, trois types de déficit en PBG désaminase ont été 
identifiés : le type le plus fréquent se caractérise par un déficit enzymatique généralisé et l'absence de protéine inactive immunologiquement décelable (cas CRM -). Un autre type se distingue par la présence dans les cellules d'enzyme immunologiquement détectable en plus ou moins grande quantité selon les familles étudiées, mais dépourvue d'activité enzymatique (cas CRM +). Enfin, dans certaines familles, le déficit enzymatique apparaît limité aux cellules non érythropoïétiques : l'activité de la PBG désaminase érythrocytaire est normale. La mutation pourrait se situer dans l'exon exprimé spécifiquement dans les cellules non érythropoïétiques ou dans le promoteur non érythropoïétique.

Porphyrie cutanée familiale et porphyrie hépatoérythrocytaire. Les études immunologiques effectuées sur l'URO décarboxylase érythrocytaire chez les patients atteints de porphyrie cutanée familiale n'ont pu mettre en évidence une protéine anormale dépourvue d'activité enzymatique $[20,22]$. A ce stade d'étude, on ne peut conclure sur le degré d'hétérogénéité de la maladie : néanmoins, l'enseignement apporté par les autres maladies métaboliques héréditaires permet de supposer que de nombreuses mutations sont probablement responsables de ces différents cas CRM -. Les recherches sur la porphyrie hépatoérythrocytaire sont plus avancées : ceci s'explique par le fait qu'il s'agit d'une transmission récessive avec atteinte des deux allèles (homozygotie vraie ou double hétérozygotie). L'hétérogénéité de la maladie a pu être mise en évidence par l'étude de quatre familles : trois mutations différentes au moins ont été détectées : un cas CRM + a été décrit ; les autres cas (CRM - ou non précisés) correspondent à une diminution du taux de synthèse de la protéine ou à une dégradation rapide de cette dernière. La caractérisation moléculaire d'un cas CRM - a été très récemment effectuée dans notre laboratoire par l'étude de la séquence de $m / s n^{\circ} 3$ vol. 3 , mars 87
l'ADNc : il s'agit d'une mutation ponctuelle en position 281 (remplacement d'une glycine par un acide glutamique) rendant la protéine très sensible aux protéases endocellulaires [23]. L'utilisation d'oligonucléotides de synthèse permettra de déterminer s'il s'agit d'un cas unique ou bien d'une mutation commune à d'autres familles atteintes d'un déficit en URO décarboxylase, qu'il s'agisse de porphyrie hépatoérythrocytaire ou de porphyrie cutanée familiale.

\section{Perspectives}

L'étude des bases métaboliques responsables des différentes porphyries bénéficie actuellement de l'introduction dans ce domaine des techniques de la biologie moléculaire. La caractérisation des mutations et de leurs conséquences fonctionnelles doit aider à la compréhension de l'expression génétique normale des gènes de la biosynthèse de l'hème.

La possibilité de suivre les mutations par l'étude de l'ADN constituera en même temps, au sein des familles atteintes de porphyries hépatiques aiguës, une aide au diagnostic des porteurs dans le cas où l'enquête familiale par dosage enzymatique s'avère non concluante. En effet, en raison de la variabilité interindividuelle des valeurs normales de l'activité enzymatique, il est impossible de distinguer entre normal et porteur sain dans environ $10 \%$ des cas. Or l'enquête familiale est fondamentale pour la prévention des crises aiguës de la maladie.

Enfin une thérapie génique spécifique à l'aide d'un rétrovirus modifié [24] est tout à fait envisageable dans les prochaines années pour les formes graves de ces maladies (porphyrie érythropoïétique congénitale et porphyrie hépatoérythrocytaire): on peut, en effet, raisonnablement penser que la correction du déficit enzymatique, au niveau de la moelle osseuse, à l'aide de cellules "greffées ", sera suffisante pour limiter la surproduction de précurseurs et/ou de porphyrines au niveau de l'organisme entier

\section{Summary}

Porphyrias are a group of disorders due to a genetic deficiency in one of the heme biosynthetic pathway enzymes. Our knowledge of the diseases has evolved from the characterization of the heme precursors, whose abnormal accumulation is responsible for the clinical manifestations of the diseases, to the determination of the enzyme deficiency specific to each porphyria. Introduction of molecular biology techniques is now allowing a better understanding of the organization of the genes encoding these finely coordinated enzymes : their genes are dispersed on various chromosomes; the underlying mechanism controlling the tissuespecific expression of two of these enzymes is under investigation (two separate genes encode ALA synthetase whereas two different promotors on the same gene can account for the two PBG-deaminase mRNAs). Acute intermittent porphyria, although a clinically homogeneous disease, is the consequence of heterogeneous mutations in the PBGdeaminase gene; a point mutation (gly $\rightarrow$ glu) in the coding sequence of the URO decarboxylase gene is responsible for the instability of the enzyme in a case of hepatoerythropoietic porphyria and further studies will show if the same mutation is responsible for the familial form of porphyria cutanea tarda (a dominant disease due to a $50 \%$ deficiency in URO decarboxylase).

\section{TIRÉS A PART}

H. de Verneuil : INSERM-U 184 et CNRSLGME, Institut de chimie biologique, Faculté de médecine, 11 , rue Humann, 67085 Strasbourg Cedex. 